\title{
VASCULAR REACTIVITY AND HIGH DIETARY EICOSAPENTAENOIC ACID
}

Warren E. Lockette, R. Clinton Webb, Brenda R. Culp and Bertram Pitt.

Departments of Physiology and Internal Medicine, University of Michigan Medical School, Ann Arbor, Michigan 48109.

\section{Abstract}

Epidemiologic studies suggest that high dietary intake of eicosapentaenoic acid (EPA), a precursor of the trienoic prostaglandins, is associated with a low incidence and reduced extent of myocardial infarction. Vascular reactivity of isolated aortic strips from rats maintained for 3 weeks on a control diet or on a diet supplemented with menhaden fish oil (17\% EPA) was examined with norepinephrine, sodium arachidonate, $\mathrm{KCl}, \mathrm{PGF}_{2} \alpha$ and nitroprusside. Aortic strips from rats fed the fish oil diet were significantly less responsive to the contractile effects of norepinephrine and arachidonate compared to those from control diet rats. Treatment of aortic strips with indomethacin decreased responsiveness to norepinephrine. The magnitude of the decrease was greater in control rats resulting in a similar vascular response between the 2 groups after blockade. Contractions to arachidonate were abolished by indomethacin. There were no differences in vascular responses to $\mathrm{KCl}, \mathrm{PGF}_{2} \alpha$ and nitroprusside in aortic strips from control diet rats and those from the $f$ ish oil diet rats. Aortic strips from the fish oil diet rats contained more EPA than those from the control diet rats. Thus, the contractile effect of norepinephrine in isolated $r$ at aortic strips is normally augmented by intrinsic prostaglandins, and this augmentation is diminished by dietary intake of EPA.

\section{Introduction}

Eicosapentaenoic acid (EPA) is found in high concentration in fish of the Atlantic Ocean, and it has been suggested that a high dietary intake of this fatty acid may be involved with the low incidence of myocardial infarction in Greenland Eskimos (1). Dietary supplementation with menhaden fish oil, an oil that is relatively high in EPA $(17 \%)$, reduces infarct size in experimental models of cerebral arterv ligation and coronary artery thrombosis $(2,3)$. These reductions in infarct size were correlated with changes in platelet aggregation. The goal of this study was to determine the effects of $f$ ish oil supplementation on vascular responsiveness in rats. 


\section{PROSTAGLANDINS}

\section{Methods}

Male Sprague-Dawley rats $(200 \mathrm{~g})$ were placed on the following diet ad libitum $(\mathrm{g} / 100 \mathrm{~g}$ diet $)$ : casein, 27.36; sucrose, 30.38 ; cornstarch, 20.49; cellulose fiber, 6.87 ; soybean oil, 6.87; AIN-76 mineral mix, 6.05; AIN-76 vitamin mix, 1.40; choline chloride, 0.23; and dl-methionine, 0.35 (AIN Ad Hoc Committee on standards for nutritional studies, 1977). The fish oil-treated rats received 16.4 $\mathrm{g} / 100 \mathrm{~g}$ of basal diet as menhaden fish oil (supplied by Dr. A. Rimbo, Zapata Haynie Co., Reedville, Virginia). After three weeks of either the control basal diet or the basal diet supplemented with fish oil, the animals were terminated and their aortae were excised. Helically cut strips of these aortae were mounted in organ chambers and isometric contractions were recorded, as described previously (4). The fatty acid content of aortic strips was determined by gas 1 iquid chromatography as described by culp et al. (3).

Vascular responses were recorded for varying concentrations of norepinephrine (Breon Laboratories, Inc.), sodium arachidonate (Sigma Chemical Co.), PGF $2 \alpha$ (Sigma Chemical Co.), PGE 2 (Sigma Chemical Co.) and nitroprusside (Roche Laboratories) added cumulatively to the muscle bath. Increasing potassium concentrations in the muscle bath were achieved by equimolar substitution of $\mathrm{NaCl}$ with $\mathrm{KCl}$. Norepinephrine, $\mathrm{PGF}_{2}$ and nitroprusside were dissolved in water; sodium arachidonate was dissolved in $100 \mathrm{mM} \mathrm{Na}{ }_{2} \mathrm{CO}_{3}$ buffer in a concentration of 1.0 $\mathrm{mg} / \mathrm{ml}$ and subsequently diluted in water. Indomethacin (Sigma Chemical Co.) was dissolved in ethanol in a concentration of 5.0 $\mathrm{mg} / \mathrm{ml}$. In the experiments requiring inhibition of prostaglandin cyclooxygenase, indomethacin was added to the muscle bath to achieve a final concentration of $5.0 \mu \mathrm{g} / \mathrm{ml}$ ten minutes prior to the addition of either norepinephrine or sodium arachidonate. Ethanol, in the concentration used in these experiments $(0.1 \%)$, had no effect on the contractile responses of the aortic strips.

\section{Results}

Cumulative addition of norepinephrine to the muscle bath produced contractile responses in aortic strips from untreated rats and fish oil-treated rats (Figure 1). Aortic strips from fish oil-treated rats were less sensitive to the catecholamine than were those from untreated rats. The concentration of norepinephrine required to produce a half-maximal response ( $D_{50}$ ) was $20.4 \mathrm{x}$ $10^{-10} \mathrm{M}$ in the aortic strips from the fish oil-treated rats compared to an ED50 of $7.4 \times 10^{-10} \mathrm{M}$ for aortic strips from untreated rats $(p<0.05)$. The values for $E D_{50}$ were determined by logit-transformation and compared statistically by Student's " $t$ " test. Treatment of aortic strips with indomethacin $(5 \mu \mathrm{g} / \mathrm{ml})$, a cyclooxygenase inhibitor, caused a significant shift to the right in the dose-response relationship to norepinephrine in aortic strips from both groups of rats. However, the magnitude of the shift was 
greater in aortic strips from untreated rats; this resulted in similar vascular responses between the two groups of rats after cvclooxygenase blockade (fish oil-treated rats ED $50=78.5 \mathrm{x}$ $10^{-10} \mathrm{M}$ versus untreated $\mathrm{rats} \mathrm{ED}_{50}=95.5 \times 10^{-10}, \mathrm{P}$ 0.05). There were no differences in the magnitude of maximal contractile responses to norepinephrine in aortic strips from either group of rats in the presence ( $\mathrm{fish}$ oil-treated rats $=613 \pm 65 \mathrm{mg}$; untreated rats $=657 \pm 80 \mathrm{mg}$ ) or absence of indomethacin ( $\mathrm{fish}$ oil-treated rats $=598 \pm 58 \mathrm{mg} ;$ untreated rats $=634 \pm \mathrm{mg})$.

Aortic strips from fish oil-treated rats were less responsive to the cumulative addition of sodium arachidonate to the muscle bath than were aortic strips from untreated rats (Figure 2). Indomethacin $(5.0 \mu \mathrm{g} / \mathrm{ml})$ completely inhibited contractile responses to sodium arachidonate in aortic strips from both groups of rats.

There were no differences in contractile responses to $\mathrm{PGF}_{2} \alpha$ (Figure 3) or depolarizing concentrations of $\mathrm{KCl}$ (Figure 4) in aortic strips from the untreated and fish oil-treated rats. The magnitude of maximal contractile responses were: 1) PGF $2 \alpha$; fish oil-treated rats $=650 \pm 94 \mathrm{mg} \cdot ;$ untreated rats $=650+110 \mathrm{mg} ; 2$ )

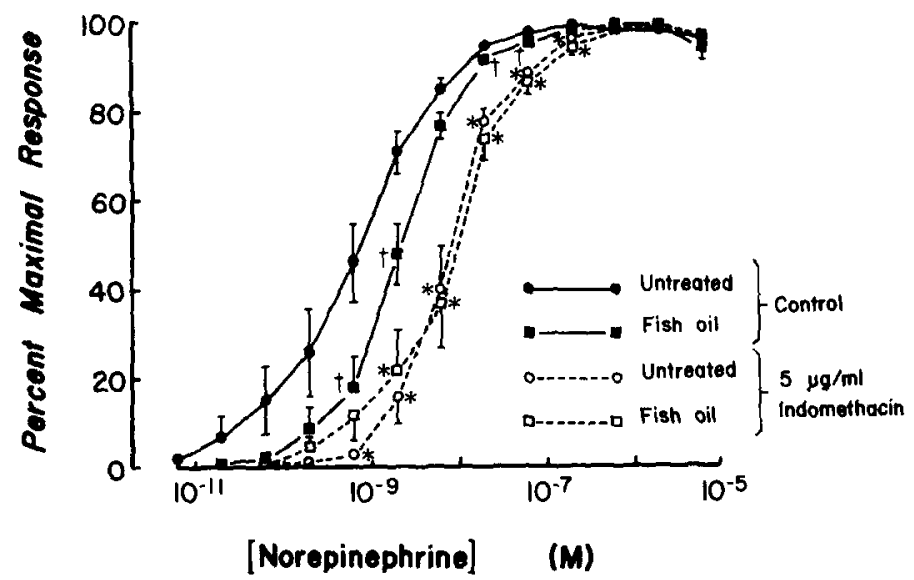

Figure 1. Dose-response to norepinephrine. Aortic strips Erom untreated and fish oil-treated rats were made to contract in response to the cumulative addition of norepinephrine to the muscle bath in the absence (contro1) and presence of $5.0 \mu \mathrm{g} / \mathrm{ml}$ indomethacin. Asterisks indicate a statistically significant difference ( $p<0.05$, Student's " $t$ " test) between the control responses and those in the presence of indomethacin. The daggers indicate a statistically significant difference between responses in aortae from untreated $r$ ats and those from fish oil-treated $r a t s$. Values are the mean \pm SEM for 6 rats in each group. 


\section{PROSTAGLANDINS}

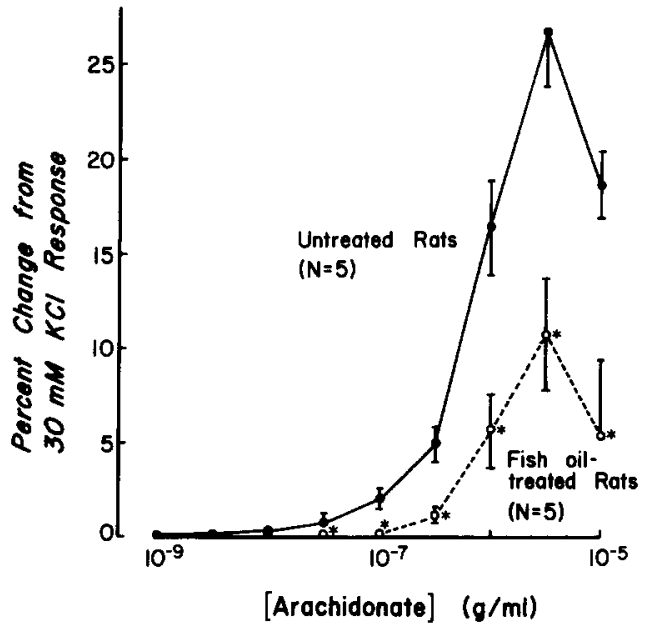

Figure 2. Dose-response to sodium arachidonate. Aortic strips from untreated and fish oil-treated rats were contracted with $30 \mathrm{mM} \mathrm{KCl}$. After the contractile response had reached a plateau, sodium arachidonate was added cumulatively to the muscle bath. Asterisks indicate a statistically significant difference between aortae from untreated rats and those from fish oil-treated rats ( $p<0.05$, Student's " $t$ " test). Values are the mean $+S E M$ for 5 rats in each group.

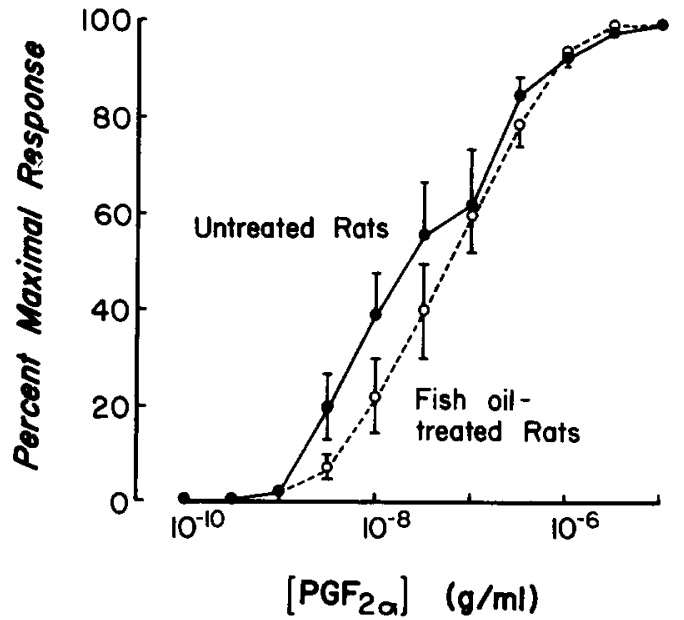

Eigure 3. Dose-response to $\mathrm{PGF}_{2} \alpha$. Aortic strips from untreated and fish oil-treated rats were made to contract in response to the cumulative addition of $\mathrm{PGF}_{2} \alpha$ to the muscle bath. Values are the mean \pm SEM for 5 rats in each group. 
$\mathrm{KC1}$; fish oil-treated $\mathrm{rats}=559 \pm 49 \mathrm{mg}$; untreated $\mathrm{rats}=505 \pm 42$ mg. Treatment of the aortic strips with PGE $_{2}$ resulted in contractions and there were no differences between untreated and $f$ ish oil-treated $r$ ats (data not shown). There was no difference in the relaxing effect of nitroprusside on aortic strips from untreated and fish oil-treated $\mathrm{rats}$ that had been contracted with $30 \mathrm{mM} \mathrm{KC} 1$ (Figure 5). Indomethacin $(5.0 \mu \mathrm{g} / \mathrm{ml})$ had no effect on the contractions induced by KC1 nor the relaxing effect of nitroprusside, suggesting that the inhibitory effect on the norepinephrine and sodium arachidonate responses was not due to a non-specific action of the drug on the smooth muscle.

The fatty acid composition of aortae from the two groups of $r a t s$ is depicted in Table 1. Aortae from the fish oil-treated rats had a higher percentage of 20:5 (n-3) (EPA), 22:5 (n-3), and 22:6 (n-3) fatty acids; these same aortae had decreased amounts of $18: 2(n-6)$ (linoleic acid), 18:1 (n-9) and 20:4 (n-6) (arachidonic acid) fatty acids compared to the aortae from untreated rats.

\section{Discussion}

These data show that a dietary supplement of menhaden fish oil decreases vascular responsiveness of isolated rat aortae to norepinephrine and sodium arachidonate, and this dietary manipulation results in a change of the fatty acid composition of the lipid fraction of blood vessel membranes.

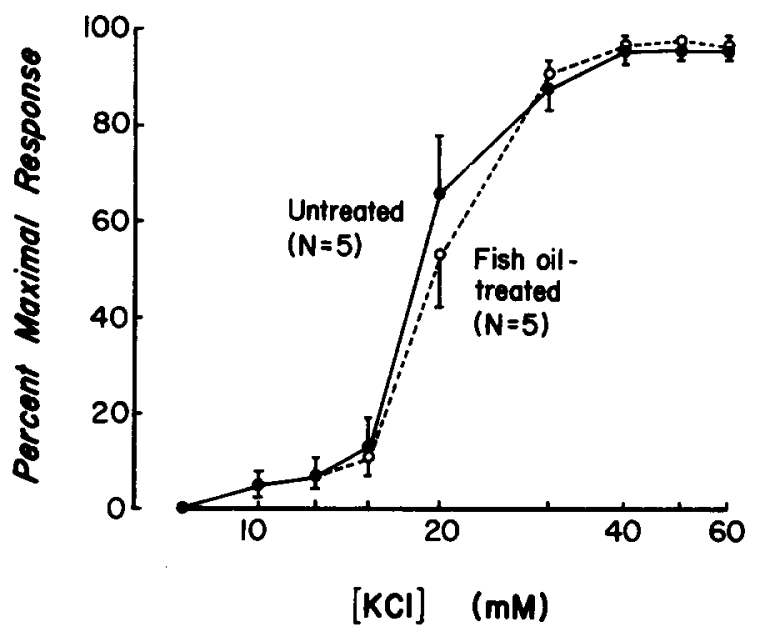

Figure 4. Dose-response to $\mathrm{KCl}$. Aortic strips from untreated and $\overrightarrow{f i s h}$ oil-treated $r$ ats were made to contract in response to increasing concentrations of $\mathrm{KC} 1$. Phentolamine $\left(10^{-6} \mathrm{M}\right)$ was added to the bath to block the effect of norepinephrine released from adrenergic nerve endings. Values are the mean \pm SEM for 5 rats in each group. 


\section{PROSTAGLANDINS}

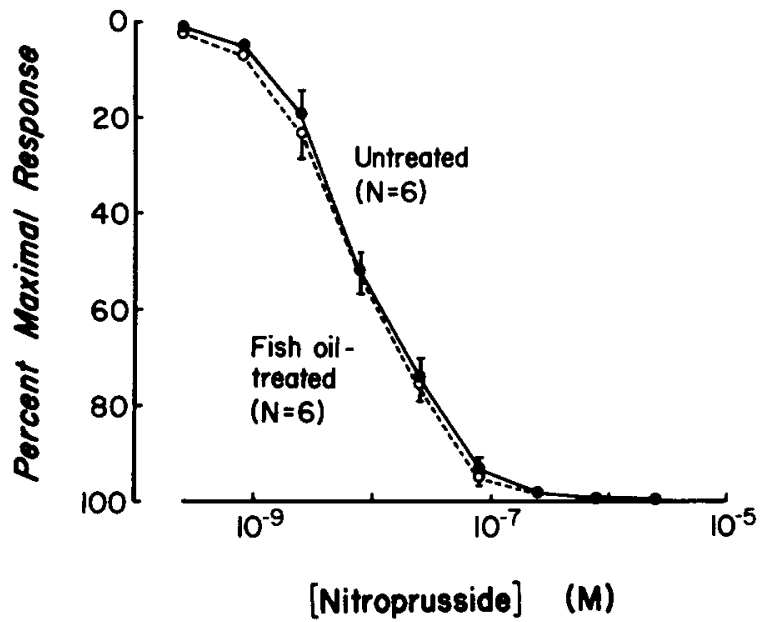

Figure 5. Dose-response to nitroprusside. Aortic strips from untreated and fish oil-treated rats were contracted with $30 \mathrm{mM} \mathrm{KC} 1$. After the contractile response had reached a plateau, nitropruside was added cumulatively to the muscle bath. Values are the mean \pm SEM for 6 rats in each group.

Table 1. Fatty acid composition of aortae

FATTY ACID

$14: 0$

$16: 0$

$16: 1(n-7)$

$1.7: 1(n-8)$

$18: 0$

$18: 1(n-9)$

$18: 2(n-6)$

$20: 1(n-9)$

$20: 2(n-6)$

$20: 3(n-6)$

$20: 4(n-6)$

$20: 5(n-6)$

$22: 4(n-6)$

$22: 5(n-6)$

$22: 5(n-3)$

$22: 6(n-3)$

\section{FISH OIL,TREATED RATS}

$5.3+0.3$

$24.4 \mp 0.6$

$7.8 \mp 0.4$

$1.1 \mp 0.0$

$8.9 \mp 1.9$

$25.2 \mp 0.8 *$

$11.5 \mp 0.9 *$

$1.9 \mp 0.2$

$0.8 \mp 0.1$

$0.2 \mp 0.1$

$2.6 \mp 0.6 \%$

$3.0 \mp 0.1 \%$

$1.5 \mp 0.0$

$0.2 \mp 0.0$

$1.6 \mp 0.1 \%$

$2.8 \mp 0.3$
UNTREATED RATS

$2.2+0.1$

$25.3 \mp 0.5$

$5.6 \mp 0.4$

$0.6 \mp 0.1$

$9.7 \mp 0.8$

$32.2 \mp 1.4$

$16.1 \mp 1.0$

$1.3 \mp 0.1$

$0.4 \mp 0.1$

$0.3 \mp 0.1$

$4.4 \mp 0.8$

$0.4 \mp 0.1$

$0.7 \mp 0.2$

$0.2 \mp 0.0$

$0.3 \mp 0.1$

$0.9 \pm 0.2$

a Fatty acids are designated by chain length:number of double bonds with the number in parentheses representing the carbon atom hetween terminal bond and methyl group. Values expressed as mole\% are mean + SEM, $n=4$ rats from each group. The asterisks indicate statistically significant differences between fish oil-treated $r a t s$ and untreated rats ( $p<0.05$, student's "t" test). 
We suggest that treatment with norepinephrine causes a release of arachidonic acid in aortic strips from untreated rats, whereas there is a simultaneous release of arachidonic acid and EPA in response to norepinephrine in aortae from fish oil-treated rats. In the aortae from untreated rats, the arachidonic acid is enzvmatically converted to the dienoic prostaglandins which contribute to the contractile responses induced by norepinephrine. In aortae from fish oil-treated rats, the simultaneous release of EPA with arachidonic acid suppresses the conversion of arachidonic acid to the dienoic prostaglandins and thereby abrogates any contributorv action of the dienoic prostaglandins on norepinephrine-induced contractions.

Whitaker et al. (5) have shown that arachidonic acid and EPA are released with equal facility in $t$ issue in response to vasoactive stimuli; also, they showed that EPA is a poor substrate for the blood vessel cvclooxygenase. It is suggested that the simultaneous release of EPA and arachidonic acid from aortae effectively blocks the conversion of arachidonic acid to vasoconstrictor metabolites. Furthermore, Whitaker et a1. found that incorporation of ${ }^{14} \mathrm{C}-\mathrm{EPA}$ into aortic rings did not lead to increased production of $\mathrm{PGI}_{3}$, suggesting that it is not the formation of trienoic prostaglandins that are responsible for the biologic activity of EPA.

Dietary manipulation of EPA intake has been tried to a limited extent in rats $(6,7)$ and humans $(8)$. Seiss et al. (8) placed seven men on a mackerel diet for one week and found a reduction in platelet aggregation and thromboxane synthesis after low dose collagen stimulation. These changes corresponded to a change in the EPA/arachidonic acid $r$ atio in platelet membranes. Seiss' group suggested that the reduction in the conversion of arachidonic acid to thromboxane was the result of competition between EPA and arachidonic acid for platelet cyclooxygenase.

The molecular mechanism by which high dietary EPA alters vascular reactivity is not known. It is not likely a result of changes in the contractile machinery (e.g., contractile proteins), as there were no differences in contractile responses to varying concentrations of $\mathrm{KCl}$ or in the relaxing effect of nitroprusside on aortic strips from untreated rats and fish oil-treated rats. It is very likely that the changes in vascular responsiveness to norepinephrine and sodium arachidonate in our study are a result of direct changes in prostaglandin metabolism for the following reasons: 1) cyclooxygenase inhibition resulted in a similar vascular response to norepinephrine in the two groups of $r$ ats;2) indomethacin inhibited vascular responses to sodium arachidonate in both groups of rats; 3 ) aortae from the fish oil-treated rats contained increased amounts of EPA $[20: 5(n-3)]$ and decreased amounts of arachidonic acid $[20: 4(n-6)\rceil$; and 4) since vascular responses to $\mathrm{PGF}_{2} \alpha$ and $\mathrm{PGE}_{2}$ were not altered, the difference in responsiveness is not due to a change in events distal to the svnthesis of prostaglandins (e.g., at receptors). This does not preclude the possible effect of other changes in cellular metabolism. For example, von Lossanyc, et al. (9) reported that a 


\section{PROSTAGLANDINS}

(a) reported that a mackerel diet in monks significantly lowered serum cholesterol and serum triglycerides and raised high density lipoprotein cholesterol in addition to changing the EPA/arachidonic acid ratio. It is not known whether these changes in serum lipids were causative or secondary to changes in prostaglandin metabolism.

Whatever the molecular mechanisms involved, these findings are significant in that thev demostrate a change in the vasculature's responsiveness to vasoactive stimuli due to a change in prostaglandin metabolism. The importance of these observations on isolated aotic strips in relation to the overall cardiovascular svstem are not clear. Generally, prostaglandins produced locally by the blood vessels reduce vascular responsiveness to norepinephrine in vivo (10). In vitro, the prostaglandins have been shown to attenuate or potentiate vascuilar responses to norepinephrine depending upon the regional vascular bed and the species studied ( 4 , $10,11)$. Kondo and associates (11) reported that $\mathrm{PGE}_{2}$ potentiated the vascular response to norepinephrine in the isolated mesenteric and hindlimb vascular beds of rats, whereas $\mathrm{PGE}_{2}$ inhibited the vascular response to norepinephrine in the splenic vascular bed. In all three vascular beds, indomethacin attenuated the vascular response to norepinephrine, and $\mathrm{PGE}_{2}$ reversed the inhibitory effect of indomethacin in mesenteric and hindlimb beds, but not in the splenic bed. Thus, it is possible that dietary manipulation of the polvunsaturated fatty acids may change vscular responsiveness, and the precise nature of the effect will be dependent upon the vascular bed studied.

\section{Acknow ledgements}

These studies were supported by grants from the Michigan Heart Association, the Michigan Memorial Phoenix Project and the National Institutes of Health (HL-27020, HL-18575). Dr. Webb is a recipient of a Research Career Development Award from the National Institutes of Hea1th (HL-00813)

\section{References}

1) Dverberg, J. and H. Bang. Hemostatic function and platelet fattv acids in Eskimos. Jancet ii: 433, 1979.

2) Black, K., B. Culp, D. Madison, 0. Randall and W. Lands. The protective effect of dietary fish oil on focal cerebral infarction. Prostaglandins and Medicine 5: 247, 1979.

3) Culp, B., W. Lands, B. Lucchesi, B. Pitt and J. Romson. The effect of dietary supplementation of fish oil on experimental mvocardial infarction. Prostaglandins 20: 1021, 1980.

4) Lockette, W.E., R.C. Webb and D.F. Bohr. Prostaglandins and potassium relaxation in vascular smooth muscle: The role of $\mathrm{Na}-\mathrm{K}$ ATPase. Circ. Res. 46: 714, 1980.

5) Whitaker, M., A. Wyche, F. Fitzpatrick, H. Sprecher and P. Needleman. Triene prostaglandins: Prostaglandin $D_{3}$ and eicosapentaenoic acid as potential antithrombotic substances. 
Proc. Nat. Acad. Sci. 76: 5919, 1979.

6) ton Hoor, F., E. de Deckere, E. Haddeman, G. Hornstra and J. Quadt. Dietary manipulation of prostaglandin and thromboxane svnthesis in aorta, heart and blood platelets of the rat. In:

Advances in Prostaglandins and Thromboxane Research, Vol. 8. (H. Samuelsson), Raven Press, New York, 1980, p. 1771.

7) Scherhag, R., H.J. Kramer and R. Dusing. Dietary administration of eicosapentaenoic and linolenic acid increases blood pressure and suppresses vascular prostacyclin synthes is in the rat. Prostaglandins 23:369. 1982.

8) Seiss, W., B. Schererm, B. Bohling, P. Roth, I. Kurtzman and P. Weber. Platelet membrane fatty acids, platelet aggregation and thromboxane formation during a mackerel diet. Lancet i: 441, 1980 .

9) von Lossanvc, T., A. Ruiter, H. Bronsgeest-Schoute, C. van Gent and $R$. Hermus. The effect of a fish diet on serum lipids in healthy human subjects. Am. J. Clin. Nutrition 31: 1340, 1978.

10) Needleman, P. and P.C. Isakson. Intrinsic prostaglandin biosynthesis in blood vessels. In: Handbook of Physiology, Sec. 2 , Vol. II. (D.F. Bohr, et al.), American Physiological Society. Bethesda, MD, 1980, P. 613 .

11.) Kondo, K., J. Misumi, T. Okuno, R. Nakamura, T. Satura and E. Kato. Effect of prostaglandin $E_{2}$ on vascular reactivity to norepinephrine in isolated rat mesenteric artery, hindlimb and splenic artery. Prostaglandins and Medicine 2: 67, 1978.

Editor: Gabor Kaley

Received: $5-28-82$

Accepted: 9-29-82 